Pacific Journal of Mathematic 


\section{METABELIAN REPRESENTATIONS OF KNOT GROUPS}

\section{RichaRd HARTLEY}

The question of determining which finite metabelian groups may be the homomorphic image of a given knot group $G$ is considered in this paper. As a starting point, it is shown that a homomorphism of a knot group onto a metabelian group $H$ such that $\left[H: H^{\prime}\right]=n$ must factor through $Z_{n} \otimes A_{n}$, where $A_{n}$ is the homology group of the $n$-fold cyclic covering space.

This is similar to a theorem of Burde [1 Satz 4], and Reyner [5] has also proven a similar result, showing in effect that such a homomorphism must factor through $Z \varnothing A_{n}$. Now, $A_{n}$ can be given the structure of a module over the ring $Z\langle t\rangle$ of $L$-polynomials, and the problem of determining the metabelian factor groups of $G$ can be reduced to determining the factor modules of $A_{n}$.

In this paper, then, a necessary and sufficient condition is given (in terms of the Alexander matrix) for a knot group to have a representation onto any given metabelian group $H$ such that $H^{\prime}$ contains no cyclic subgroup of order $n^{2}$ for any $n$. (See Theorem 1.5 and the remarks previous to Theorem 1.3.)

Such metabelian groups have a simple structure which is not shared by arbitrary metabelian groups. We therefore limit the scope of this paper to groups with this property. From Theorem 1.5 we deduce necessary and sufficient conditions for a knot group to have a representation on groups of more restricted classes in terms of the Alexander polynomial (Theorems 1.7 and 1.11).

The results obtained are similar in spirit to previous results of Fox [2] about metacyclic representations, and Riley [6] about $A_{4}$ representations, and in fact, their theorems are shown to be special cases of the theorems of this paper (see Examples 1.12 and 1.13).

In a final section it is shown how a table of the homology groups of the cyclic coverings may be used to determine the possible metabelian representations. This is included because of the ease with which one can deduce quite complete information from such a table, which may be calculated by computer. The Alexander matrix, of course, contains more information (in fact in a sense complete information) about metabelian representations, but the information therein is not so readily accessible.

I wish to thank Professor K. Murasugi for his many helpful comments concerning this paper. 
1. Throughout this paper, $K$ is a knot in $S^{3}$, and $G$, or $G(K)$, is its knot group. $G(K)$ can be written as a semi-direct product $Z Q G^{\prime}$ where $Z$ is generated by an element $t$, which is a meridian of the knot. Any cyclic group $Z$ or $Z_{n}$ will be thought of as having a distinguished generator, $t$. If $\phi$ is a homomorphism of $G$, then $G \varphi$ can be written as $Z \varphi Q G^{\prime} \varphi$. Thus, if $H$ is a metabelian factor group of $G$, then $H$ can be written as $Z_{n} Q H^{\prime}$. Note that $H$ is a semi-direct product of a cyclic group and the commutator subgroup. We make the convention that all semi-direct products considered in this paper will be of that form. It is true that if $H$ is any group with $H^{\prime}$ a finitely generated abelian group, and $H / H^{\prime} \cong C$ is cyclic, then $H$ can be written as $C Q H^{\prime}$ and $C$ acts without fixed points on $H$. Now, since $H^{\prime}$ is abelian, $\varphi$ must factor through $Z \varnothing G^{\prime} / G^{\prime \prime}$.

Given a semi-direct product $Z_{n} Q A$ with $A$ abelian, $A$ may be given the structure of a $Z\langle t\rangle$ module $(Z\langle t\rangle$ is the ring of integral $L$-polynomials) by writing $a^{t}=t^{-1} a t$, where module multiplication is indicated by writing the multiplicand as a superscript. Thus, module multiplication by $t$ is an automorphism, denoted throughout this paper by $\theta$, of order $n$. Conversely, given a $Z\langle t\rangle$ module $A$ in which $\theta$ (i.e., multiplication by $t$ ) is of order $n$, one can define a semi-direct product $Z_{n} \otimes A$. The following lemma is easily proven.

LenMa 1.1. Given groups $H=Z_{n} Q A$, and $H^{*}=Z_{m} Q A^{*}$ where $m$ divides $n$, and both $A$ and $A^{*}$ are abelian, then there is a homomorphism of $H$ onto $H^{*}$ which takes the distinguished generator $t$ of $Z_{n}$ to the distinguished generator $t^{*}$ of $Z_{m}$ if and only if $A^{*}$ is a factor module of $A$.

Note. By our convention, $A$ and $A^{*}$ are the commutator subgroups of $H$ and $H^{*}$. Thus a homomorphism of $H$ onto $H^{*}$ must take $A$ to $A^{*}$. The rest follows easily.

Now, as a $Z\langle t\rangle$ module, $G^{\prime} / G^{\prime \prime}$ is simply the knot module $\mathscr{C}$. Thus, there is a homomorphism of $G$ onto the metabelian group $Z_{n} Q A$ taking a meridian of the knot to $t$, if and only if $A$ is a factor module of the knot module. Since, for any $a$ in $A, a^{t^{n}}=a$, it follows that $A$ must be a factor module of $\mathscr{C l} / \mathbb{C}^{t^{n-1}}$, which latter module will be denoted by $A_{n}$.

THEOREM 1.2. There is a homomorphism of the knot group $G$ onto the metabelian group $Z_{n} \otimes A$ taking a meridian of $G$ to the distinguished generator of $Z_{n}$ if and only if $A$ is a factor module of $A_{n}$. 
The underlying group of $\mathscr{C}$ is the homology group of the infinite cyclic covering space of the knot, and $\theta$ is induced by a covering translation. Factoring out multiples of $t^{n}-1$ is thus equivalent to identifying every $n$th leaf of the covering. It follows that the underlying group of the module $A_{n}$ is the homology group of the $n$-fold branched cyclic covering space of the knot. Theorem 1.2 shows that $Z_{n} Q A$ is a factor group of $Z_{n} Q A_{n}$, which result is very similar to Burde [1, Satz 4]. In fact, since $Z_{n}$ acts without fixed points on $A_{n}$, it follows easily that $Z_{n}$ acts without fixed points on $A$. Thus, any metabelian factor group of a knot group is a semi-direct product of the type considered in his theorem.

Note. The condition that a meridian is mapped to the distinguished generator, $t$, results in no loss of generality of our results. For, if $G$ maps onto some group $H=Z_{n} Q H^{\prime}$, then a given meridian is mapped to a generator $t^{*}$ of some cyclic subgroup $Z_{n}^{*}$ such that $H=Z_{n}^{*} Q H^{\prime}$.

Convention $A$. When representations of a knot group $G$ onto a semi-direct product $Z_{n} Q A$ are mentioned in future, it will always be supposed implicitly that a meridian is mapped to the generator $t$ of $Z_{n}$.

It has now been shown that the search for various metacyclic factor groups of a knot group reduces to the enumeration of the factor modules of $A_{n}$.

The complicated structure of $A_{n}$ makes a complete analysis difficult. Therefore, we limit ourselves to considering factor modules $A$ of $A_{n}$, and hence semi-direct products $Z_{n} Q A$, where the group structure of $A$ is just that of an elementary abelian $p$-group ( $p$ a prime); $A=Z_{p} \oplus \cdots \oplus Z_{p}$, as a group direct sum.

In this case, $A$ must be a factor module of $A_{n} / A_{n} p$ (where $A_{n} p$ consists of all multiples of $p$ in $A_{n}$ ). This module will be denoted by $A_{n, p}$, and it may be considered as a $Z_{p}\langle t\rangle$ module, because every element is of order $p$ or 1 . Since $Z_{p}\langle t\rangle$ is a principal ideal domain (PID), this simplifies the problem greatly.

We make two remarks before we begin to examine the structure of $A_{n, p}$. Firstly, suppose that a knot group $G$ maps onto groups $Z_{n} Q A_{i} ; i=1, \cdots, r$ where $A_{i}$ is an elementary $p_{i}$ group, the $p_{i}, i=$ $1, \cdots, r$ being distinct primes. Then $G$ maps onto $Z_{n} Q\left(A_{1} \oplus A_{2} \oplus\right.$ $\left.\cdots \oplus A_{r}\right)$. To prove this, one simply observes that $Z_{n} Q\left(A_{1} \oplus \cdots\right.$ $\left.\oplus A_{k}\right)$ is the pull-back of the diagram $Z_{n} Q\left(A_{1} \oplus \cdots \oplus A_{k-1}\right) \rightarrow$ $Z_{n} \leftarrow Z_{n} Q A_{k}$. One proceeds by induction, using the results of $\S 1$ of [4]. 
Secondly, suppose that $G$ maps onto $Z_{n} Q A$ where $A$ is any group. Then $G$ maps onto $Z_{m} Q A$ for any multiple $m$ of $n$, for $Z_{m} Q A$ is a pull-back of the diagram $Z_{m} \rightarrow Z_{n} \nleftarrow Z_{n} Q A$. Furthermore, any homomorphism of $G$ onto a group $Z_{m} Q A$, where $\theta$ is of order $n<m$, may be obtained in this way by lifting from $Z_{n} Q A$. For this reason, we will call a semi-direct product $Z_{n} Q A$ nondegenerate if the order of $\theta$ is $n$.

The condition that $\theta$ be of order $n$ has an important practical consequence. In this case, $Z_{n}$ is a subgroup of $Z_{n} Q A$ which has no nontrivial subgroups which are normal in $Z_{n} Q A$. Therefore, corresponding to $Z_{n}$ there is a faithful permutation representation of $Z_{n} Q A$ as a group of permutations of the right cosets of $Z_{n}$ in $Z_{n} Q A$. Finding a faithful permutation representation is the first step in obtaining so called metabelian invariants of the knot.

The structure of a module over a PID is well known. Let $f$ be an element of $Z_{p}\langle t\rangle$ and $I(f)$ be the ideal it generates. Denote by $X_{p}(f)$ the $Z_{p}\langle t\rangle$ module $Z_{p}\langle t\rangle / I(f)$.

TheOREM 1.3. If $A$ is a $Z_{p}\langle t\rangle$-module then $A$ is a direct sum of submodules $X_{p}\left(f_{1}\right) \oplus X_{p}\left(f_{2}\right) \oplus \cdots \oplus X_{p}\left(f_{k}\right)$ where $f_{i}$ divides $f_{i+1}$. Any factor module of $A$ is isomorphic to

$$
X_{p}\left(g_{1}\right) \oplus X_{p}\left(g_{2}\right) \oplus \cdots \oplus X_{p}\left(g_{k}\right) \text { where } g_{i} \text { divides } f_{i} \text {. }
$$

Theorem 1.3 shows that to determine the factor modules of $A_{n, p}$, we need only find its direct sum decomposition.

REMARK. The module $X_{p}(f)$ is most easily realised as follows. Let $V$ be a vector space of dimension $\operatorname{deg}(f)$ over $Z_{p}$. Let $\theta$ be the automorphism of $V$ which is represented by a companion matrix for $f$ (see "Rational Canonical Form" in any text book on linear algebra). Then $V$ becomes a $Z_{p}\langle t\rangle$-module by defining $x^{t}=x \theta$, and $V$ is isomorphic to $X_{p}(f)$.

Suppose $R$ is a commutative ring with identity, and $F_{m}(R)$ is a free module with basis $\left\{e_{i}\right\}_{i=1, \cdots, m}$. Let $I$ be an ideal of $F_{m}(R)$ generated by $k$ elements, $r_{j}=\sum_{i=1}^{m} a_{j i} e_{i}$, and $A=F_{m}(R) / I$, then the matrix $\left\|a_{j i}\right\|$ is called a relation matrix for $A$ over $R$. A relation matrix for the knot module is the Alexander matrix, $M$, of the knot (according to the terminology of Rolfsen [7]). Thus, a relation matrix for $A_{n}$ is of the form $\left(\begin{array}{c}M \\ W\end{array}\right)$ where $W$ is a diagonal matrix with diagonal entries $t^{n}-1$. Let $\bmod _{p}: Z \rightarrow Z_{p}$ be the natural projection. Then $\bmod _{p}$ can be extended to a homomorphism, also called $\bmod _{p}$ from $Z\langle t\rangle$ to $Z_{p}\langle t\rangle$, and further, $\bmod _{p}$ can be applied entry by entry to a matrix over $Z\langle t\rangle$. Then it is easily seen that 
the matrix $\left(\begin{array}{c}M \\ W\end{array}\right) \bmod _{p}$ is a relation matrix for $A_{n, p}$ over $Z_{p}\langle t\rangle$. Consider the matrix $M \bmod _{p}$. Since $Z_{p}\langle t\rangle$ is a PID, this may be put in the form of a diagonal matrix, $V$, with entries $\delta_{1}(t), \delta_{2}(t), \cdots, \delta_{k}(t)$ where $\delta_{i}(t)$ divides $\delta_{i+1}(t)$. These $\delta_{i}$ will be called the mod $p$ elementary divisors of $M$. They are elements of $Z_{p}\langle t\rangle$ whose product is equal to $\Delta_{p}(t)$, the $\bmod p$ reduction of the Alexander polynomial, $\Delta(t)$. They are, of course, knot invariants.

The relation matrix for $A_{n, p}$ over $Z_{p}\langle t\rangle$ is thus of the form $\left(\begin{array}{l}V \\ W\end{array}\right)$, and this is equivalent to the diagonal matrix with diagonal entries g.c.d. $\left(\delta_{i}(t), t^{n}-1\right)$. We have proven:

THEOREM 1.4. The $Z_{p}\langle t\rangle$-module $A_{n, p}$ is a direct sum of modules $A_{n, p}=X_{p}\left(\gamma_{1}\right) \oplus X_{p}\left(\gamma_{2}\right) \oplus \cdots \oplus X_{p}\left(\gamma_{k}\right)$ where $\gamma_{i}(t)=$ g.c.d $\left(\delta_{i}(t), t^{n}-1\right)$, and the $\delta_{i}$ are the mod $p$ elementary divisors of $M$.

Note that $t-1$ does not divide $\delta_{i}$ for any $i$, for otherwise $t-1$ divides $\Delta_{p}(t)$, the $\bmod p$ Alexander polynomial. This means that $\Delta(1) \equiv 0(\bmod p)$ which is of course impossible, since $\Delta(1)= \pm 1$. This provides a proof that $\theta$ acts without fixed points on $A_{n, p}$.

Theorem 1.5. Let $H$ be an elementary abelian p-group. Then a knot group $G$ maps onto $Z_{n} Q H$ if and only if as a $Z_{p}\langle t\rangle$-module, $H=X_{p}\left(g_{1}\right) \oplus \cdots \oplus X_{p}\left(g_{k}\right)$ where $g_{i}$ divides $g_{i+1}$ for all $i$, and $g_{i}$ divides $\gamma_{i}$ for all $i$.

Note. Convention A applies. Of course, $g_{i}$ may equal 1 for some $i$, in which case $X_{p}\left(g_{i}\right)$ is just the zero module.

We deduce a simple consequence of the symmetry property of the Alexander matrix. It was shown by Torres and Fox [8] that the Alexander matrix, $M$, is equivalent to $\bar{M}^{T}$ where $M^{T}$ means the transpose of $M$, and a bar denotes conjugation. Conjugation is the map from $Z\langle t\rangle$ to $Z\langle t\rangle$ taking $t$ to $t^{-1}$. As a consequence, the $\bmod$ $p$ elementary divisors of $M$ are symmetric. That is, $\delta_{i}(t)=u \cdot \delta_{i}\left(t^{-1}\right)$ where $u$ is a unit of $Z_{p}\langle t\rangle$. We write $\delta_{i} \sim \bar{\delta}_{i}$. It follows that $\gamma_{i} \sim$ $\bar{\gamma}_{i}$. If we factorise $\gamma_{i}$ in $Z_{p}\langle t\rangle$, we see that if $f$ is a factor of $\gamma_{i}$, then so is $\bar{f}$. Thus we have:

Proposition 1.6. Let $H=X_{p}\left(g_{1}\right) \oplus \cdots \oplus X_{p}\left(g_{k}\right)$ and $\bar{H}=$ $X_{p}\left(\bar{g}_{1}\right) \oplus \cdots \oplus X_{p}\left(\bar{g}_{k}\right)$. Then a knot group maps onto $Z_{n} Q H$ if and only if it maps onto $Z_{n} Q \bar{H}$.

It is easily seen that if $f$ is of odd degree then either $t+1$ divides $f$ or $f \ngtr \bar{f}$. Now, if $n$ is odd, then $t+1$ does not divide 
$\gamma_{i}$, since if $p=2$, then $t+1$ does not divide $\delta_{i}$, and if $p>2$, then $t+1$ does not divide $t^{n}-1$. Therefore if $f$ is a divisor of $\gamma_{i}$ of odd degree then $\bar{f}$ is also a divisor and $f \nsim \bar{f}$, so the representations of $G$ onto $Z_{n} Q X_{p}(f)$ and onto $Z_{n} Q X_{p}(\bar{f})$ are distinct.

Note. If $n$ is odd, it follows from the above that each $\gamma_{i}$ is of even degree. This is to be expected in view of the result of Plans [4] that when $n$ is odd, $A_{n}$ is a direct double, that is, a direct sum of two identical abelian groups.

Theorem 1.5 allows us to find explicitly all possible metabelian groups with commutator subgroup an elementary abelian $p$-group which are factor groups of a given knot group by calculating the Alexander matrix and the mod $p$ elementary divisors. This may be a little tedious however, so the following theorem allows us to find certain factor modules of $A_{n, p}$ directly from the Alexander polynomial. The proof is easy, so it is omitted.

THEOREM 1.7. Suppose $f_{p} \in Z_{p}\langle t\rangle$ is a factor of $t^{n}-1$ which is not divisible by the square of any polynomial in $Z_{p}\langle t\rangle$. Then $G$ maps onto the group $Z_{n} \otimes X_{p}\left(f_{p}\right)$ if and only if $f_{p}$ divides $\Delta_{p}(t)$. Convention $A$ applies.

Note. (i) If $p$ does not divide $n$, then the condition that $f_{p}$ is not divisible by a square is automatically fulfilled, since then $t^{n}-1$ is a product of distinct irreducible factors in $Z_{p}\langle t\rangle$. (See below)

(ii) $Z_{n} Q X_{p}\left(f_{p}\right)$ is nondegenerate if and only if $f_{p}$ does not divide $t^{k}-1$ for any $k<n$.

We now turn aside to consider the factorization of the polynomial $t^{n}-1$ in $Z_{p}\langle t\rangle$.

Firstly, if $p$ divides $n$ and $n=m p^{\alpha}$ with g.c.d. $(m, p)=1$, then $t^{n}-1=\left(t^{m}-1\right)^{p^{\alpha}}$, thus we are reduced to the case where $p$ does not divide $m$. Now $t^{m}-1$ is a product of cyclotomic polynomials. $t^{m}-1=\prod_{i \mid m} \sigma_{i}$ where $\sigma_{i}$ is the $i$ th cyclotomic polynomial, that is, the polynomial whose roots are the primitive $i$ th roots of unity. An explicit formula for $\sigma_{i}(t)$ is

$$
\sigma_{i}(t)=\prod_{d \mid i}\left(t^{i / d}-1\right)^{\mu(d)}
$$

where $\mu(d)=0$ if $d$ is divisible by an integral square, and otherwise $\mu(d)$ equals +1 or -1 depending on whether $d$ is a product of an even or odd number of distinct primes. $\mu$ is called the Mobius function. See [9] p. 139. 
As for the factoring of the cyclotomic polynomials we have:

LEMMA 1.8. If $p$ does not divide $i$ then $\sigma_{i}$ factors in $Z_{p}\langle t\rangle$ into $\Phi(i) / s$ distinct factors of length $s$, where $s$ is the least positive integer such that $p^{s}=1(\bmod i)$, and $\Phi$ is the Euler function.

See Theorem III $12 \mathrm{E}$ in [9].

Now we examine more closely the conditions that $f_{p}$ divides $\Delta_{p}(t)$ in $Z_{p}\langle t\rangle$.

Lemma 1.9. Let $R$ be an integral domain and let $g(t)$ be a polynomial in $R[t]$. If $S$ is a matrix over $R$ with characteristic polynomial $f$, then

$$
\prod_{i=1}^{r} g\left(\xi_{i}\right)=\operatorname{det} g(S) \text { where } \xi_{1}, \cdots, \xi_{r}
$$

are the roots of $f$ in some extension of $R$.

Proof. In a suitable extension of $R$, the matrix may be put in Jordan form, in which case, the diagonal elements of $S$ are simply the $\xi_{i}$. Then the diagonal elements of $g(S)$ are $g\left(\xi_{i}\right), i=1, \cdots, r$, and $g(S)$ is upper triangular. The result follows.

LEMMA 1.10. Let $f$ be a monic polynomial in $Z[t]$ and $\Delta(t) \in$ $Z[t]$ be the Alexander polynomial of a knot $K$. Let $f_{p}$ and $\Delta_{p}(t)$ be the corresponding polynomials in $Z_{p}[t]$. Then some factor of $f_{p}$ divides $\Delta_{p}$ if and only if $p$ divides $\prod_{i=1}^{r} \Delta\left(\xi_{i}\right)$ where $\xi_{1}, \cdots, \xi_{r}$ are the roots of $f$ in the complex number field.

Proof. Let $S$ be an integral matrix with characteristic polynomial equal to $f$. Then if $\bmod _{p}$ is the natural projection of $Z$ onto $Z_{p}$, and $S_{p}=S \bmod _{p}$, then $S_{p}$ has characteristic polynomial $f_{p}$, and $(\operatorname{det} \Delta(S)) \bmod _{p}=\operatorname{det} \Delta_{p}\left(S_{p}\right)$. Now some divisor of $f_{p}$ divides $\Delta_{p}$ if and only if $\prod_{i=1}^{r} \Delta_{p}\left(\eta_{i}\right)=0$ where the $\eta_{i}$ are the roots of $f_{p}$. That is, iff det $\Delta_{p}\left(S_{p}\right)=0$, that is, iff $p$ divides $\operatorname{det} \Delta(S)=\prod_{i=1}^{r} \Delta\left(\xi_{i}\right)$.

Of course, the fact that $\Delta(t)$ is the Alexander polynomial played no part in the above proof. Combining this theorem with Theorem 1.7 we obtain

THEOREM 1.11. If $\Delta(t)$ is the Alexander polynomial of a knot $K$, then $G(K)$ maps onto $Z_{n} Q X_{p}\left(f_{p}\right)$ for some irreducible factor $f_{p}$ of $\sigma_{n}$ in $Z_{p}\langle t\rangle$, if and only if $p$ divides $\prod_{i=1}^{r} \Delta\left(\xi_{i}\right)$ where $\xi_{1}, \cdots, \xi_{r}$ 
are the primitive $n$th roots of unity. $Z_{n} Q X_{p}\left(f_{p}\right)$ is nondegenerate if and only if $p$ does not divide $n$.

EXAMPLE 1.12. Let $n=p-1$. Then $t^{n}-1$ factors as $(t-1)(t-2)$ $\cdots(t-(p-1))$ over $Z_{p}$. Therefore $G$ maps onto $Z_{p-1} Q X_{p}(t-a)=$ $\left\langle s, t ; t^{p-1}, s^{p}, t^{-1} s t=s^{a}\right\rangle$ with a meridian mapping to $t$, if and only if $t-a$ divides $\Delta_{p}(t)$. That is, if and only if $p \mid \Delta(a)$. This is a theorem of Fox [2].

EXAMPLE 1.13. If we take $n=3$ and $p=2$, then we see that $G(K)$ maps onto $Z_{3} Q\left(Z_{2} \oplus Z_{2}\right)=A_{4}$ if and only if 2 divides $\Delta(\omega)$. $\Delta\left(\omega^{2}\right)$ where $\omega$ is a primitive cube root of unity. This was previously stated but not proved by Riley [6].

In Theorem 1.11, there is nothing to tell us which of the groups $Z_{n} Q X_{p}(f)$ the knot group $G$ maps onto, in other words, which irreducible factor $f$ occurs. However as we will show right now, this is not critical, for if $f_{1}$ and $f_{2}$ are different irreducible factors of $\sigma_{n}$, then $Z_{n} Q X_{p}\left(f_{1}\right)$ and $Z_{n} Q X_{p}\left(f_{2}\right)$ are isomorphic. The isomorphism does not send the distinguished generator of $Z_{n}$ to distinguished generator and so we lose track of where a meridian is mapped. First we prove a preliminary result.

Lemma 1.14. Let $m$ be an integer not divisible by $p$ and let $f_{1}$ be one of the irreducible factors of $\sigma_{m}$ over $Z_{p}$. Let $V$ be a vector space over $Z_{p}$ and let $\theta$ be an automorphism of $V$ with minimal polynomial $f_{1}^{r}$. If $f_{2}$ is another irreducible factor of $\sigma_{m}$, then there exists an integer $s$ coprime with $m$ such that $f_{2}^{r}$ is the minimal polynomial of $\theta^{s}$.

Proof. Since an irreducible factor of $\sigma_{m}$ has no repeated roots, we may split $f_{1}^{r}$ in some splitting field to obtain $f_{1}^{r}=\left[\left(t-\xi_{1}\right) \cdots\right.$ $\left.\left(t-\xi_{k}\right)\right]^{r}$. Then if $s \neq 0$ the minimal polynomial of $\theta^{s}$ is $\prod_{j=1}^{k}\left(t-\xi_{j}^{s}\right)^{r}$. Now, since $f_{1}=\prod_{j=1}^{k}\left(t-\xi_{j}\right)$ has coefficients in $Z_{p}$, so does $\prod_{j=1}^{k}\left(t-\xi_{j}^{s}\right)$. Also, if g.c.d. $(s, m)=1$, then $\xi_{j}^{s}$ is a primitive $m$ th root of unity, since $\xi_{j}$ is a primitive $m$ th root of unity. Thus $\prod_{j=1}^{k}\left(t-\xi_{j}^{s}\right)$ must be one of the irreducible factors, $f_{i}$ of $\sigma_{m}$. Clearly, for different choices of $s$, one obtains all the possible $f_{i}$.

As an immediate consequence we obtain

THEOREM 1.15. If $\sigma_{m}^{r}$ divides $t^{n}-1$, and $f_{1}$ and $f_{2}$ are two different irreducible factors of $\sigma_{m}$ over $Z_{p}$, then $Z_{n} Q X_{p}\left(f_{1}^{r}\right)$ is isomorphic to $Z_{n} Q X_{p}\left(f_{2}^{r}\right)$. 
2. Calculation from a table of homology groups. Theorems 1.7 and 1.11 togther with the remarks about nondegenerate semidirect products allow a systematic enumeration of all the metabelian factor groups of $G$ of the form $Z_{n} Q X_{p}(f)$ where $f$ is irreducible. By pull-back techniques, one can furthermore show that if $G$ maps onto $Z_{n} Q X_{p}\left(f_{1}\right)$ and $Z_{n} Q X_{p}\left(f_{2}\right)$, where $f_{1}$ and $f_{2}$ have no common factor over $Z_{p}$, then it maps onto $Z_{n} Q X_{p}\left(f_{1} f_{2}\right)$. Thus, we can determine, from the Alexander polynomial alone, all metabelian factor groups of the form $Z_{n} Q X_{p}(f)$ where $f$ does not contain a repeated factor. (This condition is automatically fulfilled if $p \mid n$.)

If more general factor groups are desired, one must calculate the structure of $A_{n, p}$. This can be done by calculating the $\bmod p$ elementary divisors (a separate calculation for each $p$ ). An alternative method is to use a table of homology groups of the cyclic covering spaces.

If $h_{1}$ and $h_{2}$ are elements of $Z_{p}\langle t\rangle$ and g.c.d. $\left(h_{1}, h_{2}\right)=1$, then $X_{p}\left(h_{1} h_{2}\right)=X_{p}\left(h_{1}\right) \oplus X_{p}\left(h_{2}\right)$. It follows that a module over $Z_{p}\langle t\rangle$ can be broken up into a direct sum of submodules of the form $X_{p}\left(f^{\alpha}\right)$ where $f$ is irreducible.

Definition 2.1. Let $A_{n, p}$ be written as a direct $\operatorname{sum} \oplus X_{p}\left(f_{i}^{\alpha_{i}}\right)$ where the $f_{i}$ are irreducible. Let $f$ be in $Z_{p}\langle t\rangle$. Denote by $H_{n, p}(f)$ the direct sum of just those $X_{p}\left(f_{i}^{\alpha_{i}}\right)$ such that $f_{i}$ divides $f$.

Let $n=q m$ where $q=p^{\alpha}$ and g.c.d. $(m, p)=1$. Then, any irreducible factor of $t^{n}-1$ in $Z_{p}\langle t\rangle$ must divide exactly one $\sigma_{k}$ where $k \mid m$. It follows that $A_{n, p}=\bigoplus_{k \mid m} H_{n, p}\left(\sigma_{k}\right)$. However it can be deduced from Theorem 1.4 that $H_{n, p}\left(\sigma_{k}\right)=H_{k q, p}\left(\sigma_{k}\right)$. Thus:

$$
A_{n, p}=\bigoplus_{k \mid m} H_{k q, p}\left(\sigma_{k}\right) \text {. }
$$

We consider two cases.

Case 1. $p$ does not divide $n$. In this case, (2.2) becomes

$$
A_{n, p}=\bigoplus_{k \mid n} H_{k, p}\left(\sigma_{k}\right) .
$$

Thus, our goal is to determine $H_{k, p}\left(\sigma_{k}\right)$ for each $k$ dividing $n$. Since $Z_{p}$ is a field, a module $A$ over $Z_{p}\langle t\rangle$ can be considered simply as a vector space over $Z_{p}$. Define the dimension of $A$, $\operatorname{dim}(A)$ to be its dimension as a vector space. As a group, then, $A$ is a direct sum of $\operatorname{dim}(A)$ copies of $Z_{p}$. It follows from 2.3 by an easy induction that for any $n$ not divisible by $p$, 


$$
\operatorname{dim} H_{n, p}\left(\sigma_{n}\right)=\sum_{i ; n} \operatorname{dim}\left(A_{i, p}\right) \cdot \mu(n / i)
$$

where $\mu$ is the Mobius function defined previously.

$\operatorname{dim}\left(A_{i, p}\right)$ is easily read from a table of cyclic homology groups, being the largest integer $b$ such that there is a group homomorphism of $A_{i}$ onto a direct sum of $b$ copies of $Z_{p}$. If $\sigma_{n}$ is irreducible over $Z_{p}$, then $H_{n, p}\left(\sigma_{n}\right)=X_{p}\left(\sigma_{n}\right) \oplus \cdots \oplus X_{p}\left(\sigma_{n}\right)$ is completely determined by its dimension. Otherwise $\sigma_{n}$ factors into $\Phi(n) / s=r$ factors $f_{1}, \cdots, f_{r}$ of degree $s$. Then

$$
H_{n, p}\left(\sigma_{n}\right)=X_{p}\left(f_{i_{1}}\right) \oplus X_{p}\left(f_{i_{2}}\right) \oplus \cdots \oplus X_{p}\left(f_{i_{N}}\right) .
$$

The following considerations are often enough to determine the structure exactly

(i ) $N s=\operatorname{dim}\left(H_{n, p}\left(\sigma_{n}\right)\right)$.

(ii) $X_{p}\left(f_{j}\right)$ occurs at least once in this direct sum if and only if $f_{j}$ divides $\Delta_{p}(t)$ (over $Z_{p}$ ).

(iii) If $X_{p}\left(f_{j}\right)$ occurs $c$ times in this direct sum, then $f_{j}^{c}$ divides $\Delta_{p}(t)$, and the Alexander matrix has dimension at least $c \times c$.

Case 2. $p$ divides $m$.

In this case, using (2.2) it can be shown by induction that

$$
\operatorname{dim} H_{k q, p}\left(\sigma_{k}\right)=\sum_{j \mid k} \operatorname{dim}\left(A_{j q, p}\right) \cdot \mu(k / j)
$$

If $\sigma_{k}$ is irreducible over $Z_{p}$, then

$$
H_{k q, p}\left(\sigma_{k}\right)=X_{p}\left(\sigma_{k}^{\alpha_{1}}\right) \oplus X_{p}\left(\sigma_{k}^{\alpha_{2}}\right) \oplus \cdots \oplus X_{p}\left(\sigma_{k}^{\alpha_{N}}\right),
$$

with $\alpha_{i} \leqq \alpha_{i+1}$ for all $i$, and $\alpha_{N} \leqq q$. The following conditions are often sufficient to determine the $\alpha_{i}$.

(i ) $\operatorname{dim} H_{k q, p}\left(\sigma_{k}\right)=\operatorname{deg}\left(\sigma_{k}\right) \cdot \sum_{i=1}^{N} \alpha_{i}$

(ii) $N \cdot \operatorname{deg}\left(\alpha_{k}\right)=\operatorname{dim} H_{k, p}\left(\sigma_{k}\right)$.

The second condition follows from the fact that if $H_{k q, p}\left(\sigma_{k}\right)$ is of the form given above, the $H_{k, p}\left(\sigma_{k}\right)$ is a direct sum of $N$ copies of $X_{p}\left(\sigma_{k}\right)$. Thus, using (2.4) we may determine $N$. The case where $\sigma_{m}$ is not reducible may be considered using a combination of the above methods. Details are omitted.

As an example we consider the knot $9_{40}$, which is a 3 -bridged knot with Alexander polynomial $1-7 t+18 t^{2}-23 t^{3}+18 t^{4}-7 t^{5}+t^{6}$ and whose cyclic covering spaces are as follows 


\begin{tabular}{crrrrrr} 
Degree & \multicolumn{2}{c}{ Torsion } & & & & \\
2 & 5 & 15 & & & & \\
3 & 4 & 4 & 8 & 8 & & \\
4 & 3 & 3 & 3 & 15 & 15 & \\
5 & 11 & 11 & 11 & 11 & & \\
6 & 0 & 0 & 4 & 4 & 40 & 40
\end{tabular}

In explanation, the last line means that the 6 fold cyclic homology group is $Z \oplus Z \oplus Z_{4} \oplus Z_{4} \oplus Z_{40} \oplus Z_{40}$.

The following is a list of modules $H_{k q, p}\left(\sigma_{k}\right)$

$$
\begin{aligned}
& H_{2,5}\left(\sigma_{2}\right)=X_{5}\left(\sigma_{2}\right) \oplus X_{5}\left(\sigma_{2}\right) ; H_{2,3}\left(\sigma_{2}\right)=X_{3}\left(\sigma_{2}\right) \\
& H_{3,2}\left(\sigma_{3}\right)=X_{2}\left(\sigma_{3}\right) \oplus X_{2}\left(\sigma_{3}\right) \\
& H_{4,3}\left(\sigma_{4}\right)=X_{3}\left(\sigma_{4}\right) \oplus X_{3}\left(\sigma_{4}\right) ; H_{4,5}\left(\sigma_{4}\right)=0 \\
& H_{5,11}\left(\sigma_{5}\right)=X_{11}(t-5) \oplus X_{11}(t-5) \oplus X_{11}(t-9) \oplus X_{11}(t-9) \\
& H_{6,2}\left(\sigma_{3}\right)=X_{2}\left(\sigma_{3}\right) \oplus X_{2}\left(\sigma_{3}^{2}\right) ; H_{6,3}\left(\sigma_{2}\right)=X_{3}\left(\sigma_{3}^{2}\right) \\
& H_{6,5}\left(\sigma_{6}\right)=X_{5}\left(\sigma_{6}\right) .
\end{aligned}
$$

For $p \geqq 7, H_{6, p}\left(\sigma_{6}\right)=X_{p}\left(\sigma_{6}\right)$ and for $p \equiv 1 \bmod 6, X_{p}\left(\sigma_{6}\right)=X_{p}(t-\alpha) \oplus$ $X_{p}(t-\beta)$ where $\alpha$ and $\beta$ are the two roots of $\sigma_{6}$ in $Z_{p}$.

Explanation. In the case of $H_{5,11}\left(\sigma_{5}\right)$ we see that $\sigma_{5}$ factors over $Z_{11}$ into $(t-4)(t-5)(t-9)(t-3)$, of which only $t-5$ and $t-9$ divide $A_{p}(t)$. This explains the structure of $H_{5,11}\left(\sigma_{5}\right)$. As for $H_{6,2}\left(\sigma_{3}\right)$, we see that $\operatorname{dim}\left(H_{6,2}\left(\sigma_{3}\right)\right)=6$ whereas $\operatorname{dim}\left(H_{3,2}\left(\sigma_{3}\right)\right)=4$, so $H_{6,2}\left(\sigma_{3}\right)$ must be $X_{2}\left(\sigma_{3}\right) \oplus X_{2}\left(\sigma_{3}^{2}\right) . \quad H_{6,3}\left(\sigma_{2}\right)$ is treated similarly. All the others follow directly from evaluating the dimension.

Finally it seems appropriate to indicate how a given metabelian representation of a knot group is to be found. Suppose that $G$ has a representation on $Z_{n} Q A$, where $A$ is a $Z_{p}\langle t\rangle$-module of linear dimension $d$. Elements of $A$ can be written as $d$-tuples of elements of $Z_{p}$, and $\theta$ can be represented by a $d \times d$ matrix $S$ over $Z_{p}$. In particular, if $A=X_{p}(f)$ then $S$ is a companion matrix for $f$. One seeks to label the overcrossings (corresponding to Wirtinger generators) of a knot diagram with elements of $Z_{p}^{d}$ (i.e., $d$-tuples) in such way that at a crossing point with labels as shown,

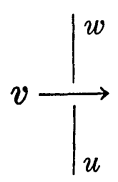

the labels obey $(u-v) S+v-w=0$. Then one verifies that if a 
Wirtinger generator corresponding to an overcrossing marked $u$ is mapped to the element $t \cdot u$ of $Z_{n} Q A$, then this is a homomorphism of the knot group.

\section{REFERENCES}

1. G. Burde, Darstellungen von Knotengruppen und eine Knoteninvariante, Hamburg Abh., 35 (1970), 107-120.

2. R. Fox, Metacyclic Invariants of Knots and Links, Canad. J. Math., 22 No. 2 (1970), 193-201.

3. R. Hartley and K. Murasugi, Homology Invariants, Canad. J. Math., 30 No. 3 (1978), 655-670.

4. A. Plans, Aportación al estudio de los grupos de homologia de los recubrimientos ciclicos ramificados correspondientes a un nudo, Revista de la Real Academia de Ciencias Exactas, Fisicas y Naturales de Madrid, 47 (1953), 161-193.

5. S. Reyner, On Metabelian and Related Invariants of Knots, Ph. D. Thesis, Princeton, 1972.

6. R. Riley, Homomorphisms of Knot groups on finite groups, Mathematics of Computation, 25, No 115 (1971), 603-619.

7. D. Rolfsen, Knots and Links, Publish or Perish, 1976.

8. G. Torres and R. H. Fox, Dual presentations of the group of a knot, Ann. of Math., 59 (1954), 211-218.

9. H. Weyl, Algebraic Theory of Numbers, Princeton, 1940.

Received August 30, 1978. This research was supported by the National Research Council of Canada.

UNIVERSITY OF TORONTO

Toronto (M5S 1A1) ONTARIo, Canada

Current address: University of Melbourne Parkville, Victoria, Australia 3052. 


\section{PACIFIC JOURNAL OF MATHEMATICS}

\section{EDITORS}

DoNALD BABBITT (Managing Editor)

University of California

Los Angeles, California 90024

HUGo Rossi

University of Utah

Salt Lake City, UT 84112

C. C. MOORE and ANDREW OGG

University of California

Berkeley, CA 94720
J. DUGUNDJI

Department of Mathematics University of Southern Californı Los Angeles, California 90007

R. Finn and J. Milgram Stanford University Stanford, California 94305

\section{ASSOCIATE EDITORS}

E. F. BECKENBACH

B. H. Neumann
F. WOLF

K. YOSHIDA

\section{SUPPORTING INSTITUTIONS}

UNIVERSITY OF BRITISH COLUMBIA CALIFORNIA INSTITUTE OF TECHNOLOGY UNIVERSITY OF CALIFORNIA MONTANA STATE UNIVERSITY UNIVERSITY OF NEVADA, RENO NEW MEXICO STATE UNIVERSITY OREGON STATE UNIVERSITY UNIVERSITY OF OREGON
UNIVERSITY OF SOUTHERN CALIFORNIA STANFORD UNIVERSITY UNIVERSITY OF HAWAII UNIVERSITY OF TOKYO UNIVERSITY OF UTAH WASHINGTON STATE UNIVERSITY UNIVERSITY OF WASHINGTON 


\section{Pacific Journal of Mathematics}

Vol. 82, No. $1 \quad$ January, 1979

Werner Bäni, Subspaces of positive definite inner product spaces of countable dimension ...................................... 1

Marilyn Breen, The dimension of the kernel of a planar set..............

Kenneth Alfred Byrd, Right self-injective rings whose essential right ideals

are two-sided

Patrick Cousot and Radhia Cousot, Constructive versions of Tarski's fixed

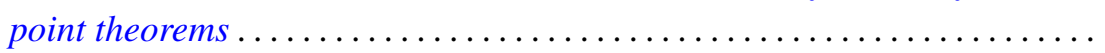

Ralph S. Freese, William A. Lampe and Walter Fuller Taylor, Congruence lattices of algebras of fixed similarity type. $I \ldots \ldots \ldots \ldots \ldots \ldots \ldots$

Cameron Gordon and Richard A. Litherland, On a theorem of Murasugi .....

Mauricio A. Gutiérrez, Concordance and homotopy. I. Fundamental

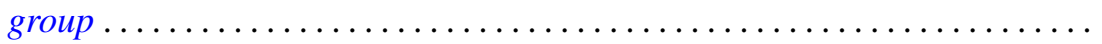

Richard I. Hartley, Metabelian representations of knot groups .............

Ted Hurley, Intersections of terms of polycentral series of free groups and free

Lie algebras ........................................

Roy Andrew Johnson, Some relationships between measures ............ 117

Oldřich Kowalski, On unitary automorphisms of solvable Lie algebras .......

Kee Yuen Lam, $K O$-equivalences and existence of nonsingular bilinear

maps...................................................

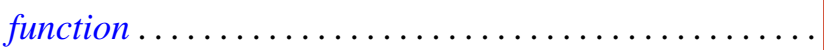

Robert A. Messer and Alden H. Wright, Embedding open 3-manifolds in compact 3-manifolds ............................

Gerald Ira Myerson, A combinatorial problem in finite fields. I . .

James Nelson, Jr. and Mohan S. Putcha, Word equations in a band of paths.

Baburao Govindrao Pachpatte and S. M. Singare, Discrete generalized Gronwall inequalities in three independent variables . .

William Lindall Paschke and Norberto Salinas, $C^{*}$-algebras associated with free products of groups ........................

Bruce Reznick, Banach spaces with polynomial norms ....

David Rusin, What is the probability that two elements of a finite group

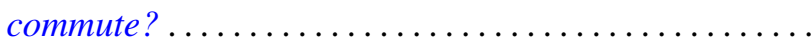

M. Shafii-Mousavi and Zbigniew Zielezny, On hypoelliptic differential operators of constant strength ...

Joseph Gail Stampfli, On selfadjoint derivation ranges .... . . .

Robert Charles Thompson, The case of equality in the matrix-valued triangle

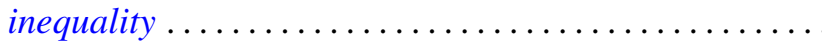

Marie Angela Vitulli, The obstruction of the formal moduli space in the negatively graded case. 